\title{
A rigorous scattering approach to quasifree fermionic systems out of equilibrium
}

\author{
Walter H. Aschbacher* \\ Université de Toulon, Aix Marseille Univ, CNRS, CPT, Toulon, France
}

\begin{abstract}
Within the rigorous axiomatic framework for the description of quantum mechanical systems with a large number of degrees of freedom, we construct the so-called nonequilibrium steady state for the quasifree fermionic system corresponding to the isotropic $X Y$ chain in which a finite sample, subject to a local gauge breaking anisotropy perturbation, is coupled to two thermal reservoirs at different temperatures. Using time dependent and stationary scattering theory, we rigorously prove, from first principles, that the nonequilibrium system under consideration is thermodynamically nontrivial, i.e., that its entropy production rate is strictly positive.
\end{abstract}

MSC (2010) 46L60, 47A40, 47B15, 82C10, 82C23

PACS (2010) 02.30.Tb, 03.65.Nk, 71.10.Ca, 72.10.Fk, 65.40.gd

Keywords Open systems, nonequilibrium quantum statistical mechanics, quasifree fermions, Hilbert space scattering theory, nonequilibrium steady state, entropy production.

\section{Introduction}

In recent years, a wide range of important thermodynamic properties of open quantum systems have successfully been derived from first principles. A precise analysis of such systems having a large, i.e., often, in physically idealized terms, an infinite number of degrees of freedom, is most clearly carried out within the axiomatic framework of operator algebras. As a matter of fact, after having been heavily used in the 1960s, in particular for the description of quantum systems in thermal equilibrium (see, for example, [8]), the benefits of this framework have again started to unfold more recently in the physically much more general situation of open quantum systems out of equilibrium. In the latter field, most of the rare mathematically rigorous results have been obtained for the so-called nonequilibrium

\footnotetext{
*walter.aschbacher@univ-tln.fr
} 
steady states (NESSs) introduced in [13] by means of scattering theory on the algebra of observables.

In quantum statistical mechanics both in and out of equilibrium, an important role is played by the so-called quasifree fermionic systems, and this is true not only because of their mathematical accessibility but also when it comes to real physical applications. Indeed, from a mathematical point of view, these systems allow for a simple and powerful description by means of scattering theory restricted to the underlying 1-particle Hilbert space over which the fermionic algebra of observables is constructed. The restriction of the dynamics to the 1-particle sector opens the way for a rigorous mathematical analysis of many properties which are of fundamental physical interest. But, beyond their importance due to their mathematical accessibility, quasifree fermions also constitute a class of systems which effectively describe nature. Aside from the various electronic systems in their independent electron approximation, they also play a part in the rigorous approach to spin systems. One of the most prominent representatives of the latter is the so-called XY spin chain, introduced in 1961 in [11], for which a physical realization has already been identified in the late 1960s (see, for example, [9]). Its impact on the interplay between the experimental, numerical, theoretical, and mathematical research activity in the field of low-dimensional magnetic systems is being felt ever since (see, for example, [12]).

In the present paper, we rigorously analyze, from first principles, the entropy production rate in the quasifree fermionic system over the two-sided discrete line $\mathbb{Z}$ which, in the spin picture, corresponds to the isotropic $X Y$ spin chain perturbed by a local anisotropy (in contrast to [6], where the anisotropy acts as a global and homogeneous perturbation).

In order to specify the desired nonequilibrium configuration, we first fix $n \in \mathbb{Z}$ with $n \geq 1$ and cut the finite piece

$$
\mathbb{Z}_{S}:=\{x \in \mathbb{Z}|| x \mid \leq n\}
$$

of length $2 n+1$ out of the two-sided discrete line. This piece plays the role of the configuration space of the confined sample, whereas the remaining infinite parts,

$$
\begin{aligned}
& \mathbb{Z}_{L}:=\{x \in \mathbb{Z} \mid x \leq-(n+1)\}, \\
& \mathbb{Z}_{R}:=\{x \in \mathbb{Z} \mid x \geq n+1\},
\end{aligned}
$$

act as the configuration spaces of the extended thermal reservoirs. Over these configuration spaces, we define the initial state to be the decoupled product of three thermal equilibrium states carrying the corresponding inverse temperatures

$$
0=\beta_{S}<\beta_{L}<\beta_{R}<\infty .
$$

The NESS is then constructed with respect to the full time evolution which, by definition, not only couples the sample to the reservoirs but also exposes the sample to a local anisotropy perturbation of strength

$$
\gamma \in \mathbb{R},
$$


whose support resides on the sites $\{a, a+1\}$, where $a \in \mathbb{Z}$ satisfies

$$
-n \leq a \leq n-1 .
$$

The paper is organized as follows.

Section 2 specifies the nonequilibrium setting we are interested in, i.e., it introduces the canonical anticommutation relation (CAR) algebra of observables, its selfdual version, the quasifree dynamics generated by the 1-particle Hamiltonians, and the quasifree initial state.

Section 3 is devoted to the definition and the construction of the NESS in the nonequilibrium setting at hand. It turns out that the absolutely continuous part of its 2-point operator, computed by means of time dependent scattering theory, is determined through the so-called intermediate wave operator which, by definition, compares the free isotropic $X Y$ dynamics with the free isotropic $\mathrm{XY}$ dynamics perturbed by a local anisotropy.

Section 4 contains the derivation of the action of the intermediate wave operator using stationary scattering theory. Due to the fact that the local anisotropy is a 2-site perturbation breaking gauge invariance, the action of the intermediate wave operator is substantially more complicated than the one from [5] for a gauge invariant local 1-site perturbation.

Section 5 introduces the notions of heat flux and entropy production rate. A general formula is derived for the NESS expectation value of the extensive energy current observable describing the energy flow through the sample as a function of the strength of the anisotropy perturbation. It is proven that the nonequilibrium system under consideration is thermodynamically nontrivial, i.e., that its entropy production rate, the first fundamental physical quantity for systems out of equilibrium, is strictly positive.

\section{Nonequilibrium setting}

In this section, we specify the nonequilibrium setting we are interested in. First recall that, in the operator algebraic formalism of quantum statistical mechanics, a physical system is characterized by an algebra of observables, by a group of time evolution automorphisms, and by a normalized positive linear state functional on the observable algebra (see, for example, [8] for a detailed description of this formalism). Definitions 1, 4, and 8 below spell out the corresponding three ingredients for the quasifree nonequilibrium setting to be studied. Here and there, we will also make brief remarks on the underlying general framework.

In the following, for all complex Hilbert spaces $\mathcal{H}$, we denote by $\mathcal{L}(\mathcal{H})$ and $\overline{\mathcal{L}}(\mathcal{H})$ the sets of bounded linear and antilinear operators on $\mathcal{H}$, respectively. Moreover, $\mathcal{L}^{0}(\mathcal{H})$ stands for the finite rank operators and $\mathcal{L}^{1}(\mathcal{H})$ for the trace class operators on $\mathcal{H}$. For elements $A, B$ in the various sets in question below, the commutator and the anticommutator of $A$ and $B$ are denoted by $[A, B]:=A B-B A$ and $\{A, B\}:=A B+B A$, respectively. Finally, $\sigma_{1}, \sigma_{2}, \sigma_{3} \in \mathbb{C}^{2 \times 2}$ are the usual Pauli matrices and, for all $m \in \mathbb{N}$, we denote by $\mathbb{C}^{m \times m}$ the complex $m \times m$ matrices. 


\section{Definition 1 (Observables)}

(a) 1-particle Hilbert space

Let $\mathbb{Z}$ be the configuration space of the system and let

$$
\mathfrak{h}:=\ell^{2}(\mathbb{Z})
$$

be the separable complex 1-particle Hilbert space of square-summable complex-valued functions on $\mathbb{Z}$. Moreover, we set $\mathfrak{H}:=\mathfrak{h} \oplus \mathfrak{h}$ and, on both $\mathfrak{h}$ and $\mathfrak{H}$, we denote the scalar products and the corresponding induced norms by $(\cdot, \cdot)$ and $\|\cdot\|$, respectively.

(b) Algebra of observables

The algebra of observables is defined to be the CAR algebra over $\mathfrak{h}$, denoted by

$$
\mathfrak{A}:=\operatorname{CAR}(\mathfrak{h}),
$$

whose generators are written, as usual, as $1, a(f)$, and $a^{*}(f)$ for all $f \in \mathfrak{h}$.

(c) Selfdual generators

The complex linear map $B: \mathfrak{H} \rightarrow \mathfrak{A}$, defined, for all $F:=f_{1} \oplus f_{2} \in \mathfrak{H}$, by

$$
B(F):=a^{*}\left(f_{1}\right)+a\left(\zeta f_{2}\right),
$$

where $\zeta f:=\bar{f}$ stands for the complex conjugation on $\mathfrak{h}$, satisfies the relations

$$
\begin{aligned}
B^{*}(F) & =B(\Gamma F), \\
\left\{B^{*}(F), B(G)\right\} & =(F, G) 1,
\end{aligned}
$$

where the antiunitary involution $\Gamma \in \overline{\mathcal{L}}(\mathfrak{H})$ is defined to act as the operator matrix $\Gamma:=\zeta \sigma_{1}$ on the direct sum $\mathfrak{H}=\mathfrak{h} \oplus \mathfrak{h}$, and (11), called the selfdual CARs, follows from the usual CARs.

(d) Selfdual second quantization

The complex linear map $b: \mathcal{L}^{0}(\mathfrak{H}) \rightarrow \mathfrak{A}$, defined, for all $m \in \mathbb{N}$, all $F_{1}, \ldots, F_{m} \in \mathfrak{H}$, all $G_{1}, \ldots, G_{m} \in \mathfrak{H}$, and $A:=\sum_{i=1}^{m}\left(F_{i}, \cdot\right) G_{i}$, by

$$
b(A):=\sum_{i=1}^{m} B\left(G_{i}\right) B^{*}\left(F_{i}\right),
$$

is called the selfdual second quantization of $A$.

Remark 2 The algebra of observables $\mathfrak{A}$ is a so-called $C^{*}$-algebra. It is $*$-isomorphic to $\overline{\operatorname{SDC}}(\mathfrak{H}, \Gamma)$, the $\left(C^{*}\right.$-completed) selfdual CAR algebra over $\mathfrak{H}$ and $\Gamma$. The selfdual framework is a useful general concept which has been developed in [3] and [1] (see there for a more detailed description of the selfdual objects used in the following).

Remark 3 Note that (12) does not depend on the choice of the functions $F_{1}, \ldots, G_{m}$ which represent $A$. Moreover, the definition of $b$ can be extended to $\mathcal{L}^{1}(\mathfrak{H})$ using the fact that $\mathcal{L}^{0}(\mathfrak{H})$ is dense in $\mathcal{L}^{1}(\mathfrak{H})$ with respect to the so-called trace norm. 
We next specify the 2nd ingredient. As discussed in the introduction, the Hamiltonians which we will introduce describe the decoupling of the reservoirs from the sample and the coupled system with and without the local anisotropy perturbation.

In the following, the completely localized elements of the orthonormal Kronecker basis $\left\{\delta_{x}\right\}_{x \in \mathbb{Z}}$ of $\mathfrak{h}$ are given, for all $x, y \in \mathbb{Z}$, by $\delta_{x}(y):=1$ if $y=x$ and $\delta_{x}(y):=0$ if $y \neq x$. Moreover, for all $A \in \mathcal{L}(\mathcal{H})$, we will use the notation $\operatorname{Re}[A]:=\left(A+A^{*}\right) / 2$ and $\operatorname{Im}[A]:=\left(A-A^{*}\right) /(2 \mathrm{i})$.

\section{Definition 4 (Dynamics)}

(a) 1-particle Hamiltonians

Using the right translation $u \in \mathcal{L}(\mathfrak{h})$ and the localization operator $p_{x, y} \in \mathcal{L}(\mathfrak{h})$, given by $(u f)(x):=f(x-1)$ and $p_{x, y} f:=f(x) \delta_{y}$ for all $f \in \mathfrak{h}$ and all $x, y \in \mathbb{Z}$, we define

$$
\begin{aligned}
h & :=\operatorname{Re}[u], \\
v_{\mathrm{d}} & :=\operatorname{Re}\left[u^{-n} p_{0,0} u^{n+1}\right]+\operatorname{Re}\left[u^{n+1} p_{0,0} u^{-n}\right], \\
v & :=\operatorname{Im}\left[p_{a+1, a}\right] .
\end{aligned}
$$

The liftings to $\mathcal{L}(\mathfrak{H})$ are given by $H:=h \sigma_{3}, V_{\mathrm{d}}:=v_{\mathrm{d}} \sigma_{3}, H_{\mathrm{d}}:=H-V_{\mathrm{d}}$, and

$$
\begin{aligned}
V & :=v \sigma_{2}, \\
H_{\gamma} & :=H+\gamma V .
\end{aligned}
$$

The Hamiltonians $H$ and $H_{\mathrm{d}}$, diagonal with respect to $\mathfrak{H}=\mathfrak{h} \oplus \mathfrak{h}$, are called the $X Y$ Hamiltonian and the decoupled Hamiltonian, respectively, whereas $H_{\gamma}$ is non-diagonal and called the anisotropy Hamiltonian.

(b) Dynamics

The quasifree dynamics generated by the XY Hamiltonian, the decoupled Hamiltonian, and the anisotropy Hamiltonian are defined, for all $t \in \mathbb{R}$ and all $F \in \mathfrak{H}$, by

$$
\begin{aligned}
\tau^{t}(B(F)) & :=B\left(\mathrm{e}^{\mathrm{i} t H} F\right), \\
\tau_{\mathrm{d}}^{t}(B(F)) & :=B\left(\mathrm{e}^{\mathrm{i} t H_{\mathrm{d}}} F\right), \\
\tau_{\gamma}^{t}(B(F)) & :=B\left(\mathrm{e}^{\mathrm{i} t H_{\gamma}} F\right),
\end{aligned}
$$

and by a suitable extension to the whole of $\mathfrak{A}$ (see Remark 5). The dynamics $\tau^{t}, \tau_{\mathrm{d}}^{t}$, and $\tau_{\gamma}^{t}$ are called the $X Y$ dynamics, the decoupled dynamics, and the anisotropy dynamics, respectively.

Remark 5 In the selfdual framework, an operator $A \in \mathcal{L}(\mathfrak{H})$ is called a Hamiltonian if $A^{*}=A$ and $\Gamma A \Gamma=-A$. The second condition is a consequence of (10), of the fact that, by definition, the dynamics $\sigma^{t}$ generated by $A$ as in (18) - (20) is, for all $t \in \mathbb{R}$, a $*$-automorphism on $\mathfrak{A}$, i.e., a map from $\mathfrak{A}$ to $\mathfrak{A}$ preserving the vector space structure, the algebra multiplication, and the $*$-operation on $\mathfrak{A}$ (we will denote by $\operatorname{Aut}(\mathfrak{A})$ the set of all such maps), and of $\|F\| / \sqrt{2} \leq$ $\|B(F)\| \leq\|F\|$ (where $\|B(F)\|$ is the $C^{*}$-norm of $B(F) \in \mathfrak{A}$ ). Both conditions are satisfied for all the Hamiltonians of Definition 4(b).

In addition, the dynamics $\left\{\sigma^{t}\right\}_{t \in \mathbb{R}} \subseteq \operatorname{Aut}(\mathfrak{A})$ is defined to be a strongly continuous group, i.e., the map $\mathbb{R} \ni t \mapsto \sigma^{t} \in \operatorname{Aut}(\mathfrak{A})$ is a group homomorphism and, for all $A \in \mathfrak{A}$, the map 
$\mathbb{R} \ni t \mapsto \sigma^{t}(A) \in \mathfrak{A}$ is continuous with respect to the $C^{*}$-norm on $\mathfrak{A}$. Such a pair $\left(\mathfrak{A}, \sigma^{t}\right)$ is sometimes called a $C^{*}$-dynamical system.

Remark 6 Defining parity $\theta \in \mathcal{L}(\mathfrak{h})$ and the local gauge transformation $\xi \in \mathcal{L}(\mathfrak{h})$ by $(\theta f)(x):=$ $f(-x)$ and $(\xi f)(x):=\mathrm{e}^{\mathrm{i} \pi x} f(x)$ for all $f \in \mathfrak{h}$ and all $x \in \mathbb{Z}$, we get the symmetries

$$
\begin{aligned}
{[h, u] } & =0, \\
{[h, \theta] } & =0, \\
\{h, \xi\} & =0,
\end{aligned}
$$

which we will use below in the proof of Proposition 16.

Remark 7 The model specified by Definition 4 has its origin in the XY model whose Hamiltonian density has the form

$$
(1+\gamma) \sigma_{1}^{(x)} \sigma_{1}^{(x+1)}+(1-\gamma) \sigma_{2}^{(x)} \sigma_{2}^{(x+1)},
$$

where the superscripts denote the sites in $\mathbb{Z}$ of the local Hilbert space of the spin chain on which the Pauli matrices act. Indeed, using the so-called Araki-Jordan-Wigner transformation introduced in [2] for 1-dimensional systems whose configuration space extends infinitely in both directions, (24), in the fermionic picture, reads (up to a global prefactor)

$$
a_{x}^{*} a_{x+1}+a_{x+1}^{*} a_{x}+\gamma\left(a_{x}^{*} a_{x+1}^{*}+a_{x+1} a_{x}\right),
$$

where we set $a_{x}:=a\left(\delta_{x}\right)$ and $a_{x}^{*}:=a^{*}\left(\delta_{x}\right)$ for all $x \in \mathbb{Z}$.

In order to treat the anisotropic case $\gamma \neq 0$, i.e., the case in which there is an asymmetry between the 1 st and the 2 nd term in (24), the selfdual quasifree setting is most natural since gauge invariance is broken in (25). Hence, due to the presence of the $\gamma$-term, the anisotropy Hamiltonian acquires non-diagonal components with respect to $\mathfrak{H}=\mathfrak{h} \oplus \mathfrak{h}$ (see (16) and (17)). In many respects, the truly anisotropic $X Y$ model is substantially more complicated than the isotropic one.

We now arrive at the specification of the initial state, the 3rd and last ingredient needed for the construction of the NESS we are interested in. As discussed in the introduction, it describes the initial configuration in which the left and right reservoirs are decoupled from the sample.

In the following, for all $M \subseteq \mathbb{R}$, we denote by $1_{M}$ the usual characteristic function of $M$ on $\mathbb{R}$, i.e., $1_{M}(x)$ equals 1 if $x \in M$ and is 0 otherwise. Moreover, recall again that a state $\omega$ is a normalized positive linear functional on the observable algebra $\mathfrak{A}$, and let us denote by $\mathcal{E}_{\mathfrak{A}}$ the set of all states.

\section{Definition 8 (Initial state)}

(a) Fermi-Dirac function

For all $\beta \in \mathbb{R}$ and all $e \in \mathbb{R}$, the Fermi-Dirac function is defined by

$$
\rho_{\beta}(e):=\frac{1}{1+\mathrm{e}^{\beta e}} .
$$




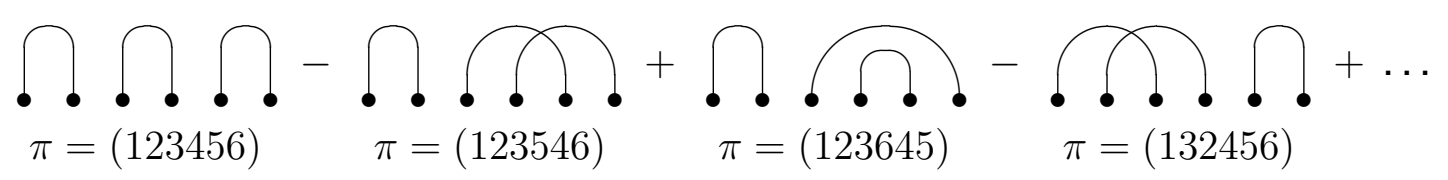

Figure 1: Some of the pairings for $m=3$. The number of intersections $I$ per graph relates to the signature of the corresponding permutation $\pi$ as $\operatorname{sign}(\pi)=(-1)^{I}$.

(b) Projected 1-particle Hamiltonians

With the help of the orthogonal projections $p_{L}, p_{R} \in \mathcal{L}(\mathfrak{h})$, given by $p_{L} f:=1_{\mathbb{Z}_{L}} f$ and $p_{R} f:=$ $1_{\mathbb{Z}_{R}} f$ for all $f \in \mathfrak{h}$, the 1-particle Hamiltonians $h_{L}, h_{R} \in \mathcal{L}(\mathfrak{h})$ are defined by

$$
\begin{aligned}
& h_{L}:=p_{L} h p_{L}, \\
& h_{R}:=p_{R} h p_{R},
\end{aligned}
$$

and their liftings to $\mathcal{L}(\mathfrak{H})$ by $H_{L}:=h_{L} \sigma_{3}$ and $H_{R}:=h_{R} \sigma_{3}$.

(c) Initial state

We define the initial state $\omega_{\mathrm{d}} \in \mathcal{E}_{\mathfrak{A}}$ to be the decoupled quasifree state whose 2-point operator $S_{\mathrm{d}} \in \mathcal{L}(\mathfrak{H})$ has the form

$$
\begin{aligned}
S_{\mathrm{d}} & :=\left(1-s_{\mathrm{d}}\right) \oplus \zeta s_{\mathrm{d}} \zeta, \\
s_{\mathrm{d}} & :=\rho_{1}\left(\beta_{L} h_{L}+\beta_{R} h_{R}\right),
\end{aligned}
$$

where the operator $s_{\mathrm{d}} \in \mathcal{L}(\mathfrak{h})$ is defined with the help of the spectral theorem.

Remark 9 In the selfdual framework, an operator $S \in \mathcal{L}(\mathfrak{H})$ is called a 2-point operator if $S^{*}=S, 0 \leq S \leq 1$, and $\Gamma S \Gamma=1-S$ (the last condition comes, in particular, from (10), (11), and (31) below). For any (not necessarily quasifree) state $\omega \in \mathcal{E}_{\mathfrak{A}}$, there exists a unique 2-point operator $S$ such that, for all $F, G \in \mathfrak{H}$, it holds that

$$
\omega\left(B^{*}(F) B(G)\right)=(F, S G) .
$$

If $\omega$ is a quasifree state induced by the 2-point operator $S$, it is completely characterized by its 2-point function (31) since, by definition, $\omega$ is even and the nonvanishing many-point functions factorize in Pfaffian form, i.e., for all $m \in \mathbb{N}$ and all $F_{1}, \ldots, F_{2 m} \in \mathfrak{H}$, we have

$$
\omega\left(B\left(F_{1}\right) \ldots B\left(F_{2 m}\right)\right)=\operatorname{pf}\left(\left[\left(\Gamma F_{i}, S F_{j}\right)\right]_{i, j=1}^{2 m}\right),
$$

and we recall that the Pfaffian is defined by $\operatorname{pf}(A):=\sum_{\pi} \operatorname{sign}(\pi) \prod_{i=1}^{m} A_{\pi(2 i-1), \pi(2 i)}$ for all $A \in$ $\mathbb{C}^{2 m \times 2 m}$, where the sum is running over all the $(2 m) ! /\left(2^{m} m !\right)$ pairings of the set $\{1, \ldots, 2 m\}$, i.e., over all the permutations $\pi$ in the permutation group of $2 m$ elements satisfying $\pi(2 i-1)<$ $\pi(2 i+1)$ for all $i \in\{1, \ldots, m-1\}$ and $\pi(2 i-1)<\pi(2 i)$ for all $i \in\{1, \ldots, m\}$ (see Figure 1). 


\section{Nonequilibrium steady state}

In this section, we give a precise definition of the NESS discussed in the introduction.

Definition 10 (NESS) The state $\omega_{\gamma} \in \mathcal{E}_{\mathfrak{A}}$, defined, for all $A \in \mathfrak{A}$, by

$$
\omega_{\gamma}(A):=\lim _{T \rightarrow \infty} \frac{1}{T} \int_{0}^{T} \mathrm{~d} t \omega_{\mathrm{d}}\left(\tau_{\gamma}^{t}(A)\right),
$$

is called the anisotropy NESS associated with the initial state $\omega_{\mathrm{d}}$ and the anisotropy dynamics $\tau_{\gamma}^{t}$. Moreover, its 2-point operator is denoted by $S_{\gamma} \in \mathcal{L}(\mathfrak{H})$.

Remark 11 The general definition, which we already specialized to our case in Definition 10 (we will see below that the limit in (33) exists), stems from [13] and defines the NESSs to be the limit points in the weak-* topology of the net defined by the ergodic mean between 0 and $T>0$ of the given initial state time evolved by the perturbed dynamics of interest (note that, due to the Banach-Alaoglu theorem, the set of such NESSs is not empty). In general, the averaging procedure allows to treat a nonvanishing contribution to the point spectrum of the Hamiltonian which generates the full time evolution.

In the following, for all selfadjoint operators $A \in \mathcal{L}(\mathfrak{H})$, we denote by $1_{\mathrm{ac}}(A), 1_{\mathrm{sc}}(A)$, and $1_{\mathrm{pp}}(A)$ the orthogonal projections onto the absolutely continuous subspace, the singular continuous subspace, and the pure point subspace of $A$, respectively. Moreover, eig $(A)$ stands for the set of eigenvalues of $A$, and $1_{e}(A)$ denotes the spectral projection onto the eigenspace associated with the eigenvalue $e \in \operatorname{eig}(A)$. Furthermore, the limit with respect to the strong operator topology on $\mathcal{L}(\mathfrak{H})$ is written as $\mathrm{s}-\lim$.

The main objects for our scattering approach are the wave operators which are defined as follows.

Definition 12 (Wave operators) The operators $W_{\mathrm{d}, \gamma}, W_{\mathrm{d}}, W_{\gamma} \in \mathcal{L}(\mathfrak{H})$, defined by

$$
\begin{aligned}
W_{\mathrm{d}, \gamma} & :=\mathrm{s}-\lim _{t \rightarrow \infty} \mathrm{e}^{-\mathrm{i} t H_{\mathrm{d}}} \mathrm{e}^{\mathrm{i} t H_{\gamma}} 1_{\mathrm{ac}}\left(H_{\gamma}\right), \\
W_{\mathrm{d}} & :=\mathrm{s}-\lim _{t \rightarrow \infty} \mathrm{e}^{-\mathrm{i} t H_{\mathrm{d}}} \mathrm{e}^{\mathrm{i} t H}, \\
W_{\gamma} & :=\mathrm{s}-\lim _{t \rightarrow \infty} \mathrm{e}^{-\mathrm{i} t H} \mathrm{e}^{\mathrm{i} t H_{\gamma}} 1_{\mathrm{ac}}\left(H_{\gamma}\right),
\end{aligned}
$$

are called the anisotropy wave operator, the $X Y$ wave operator, and the intermediate wave operator, respectively.

Remark 13 The Kato-Rosenblum theorem from scattering theory for perturbations of trace class type guarantees the existence (and completeness) of (34), (35), and (36) (see, for example, [7] or [14]). Indeed, (14) and (15) imply that $V_{\mathrm{d}}, V \in \mathcal{L}^{0}(\mathfrak{H})$ and, hence, all the differences between the corresponding Hamiltonians satisfy $H_{\mathrm{d}}-H_{\gamma}, H_{\mathrm{d}}-H, H-H_{\gamma} \in \mathcal{L}^{1}(\mathfrak{H})$. Moreover, note that $1_{\mathrm{ac}}(H)=1$ since $h$ is the Laplacian on the discrete line (see also the proof of Proposition 16 below). 
In the next theorem, we determine the 2-point operator of the anisotropy NESS.

Theorem 14 (NESS 2-point operator) The anisotropy NESS $\omega_{\gamma}$ associated with the initial state $\omega_{\mathrm{d}}$ and the anisotropy dynamics $\tau_{\gamma}^{t}$ exists and its 2-point operator has the form

$$
S_{\gamma}=W_{\mathrm{d}, \gamma}^{*} S_{\mathrm{d}} W_{\mathrm{d}, \gamma}+\sum_{e \in \operatorname{eig}\left(H_{\gamma}\right)} 1_{e}\left(H_{\gamma}\right) S_{\mathrm{d}} 1_{e}\left(H_{\gamma}\right)
$$

Proof.

(a) Evolution matrix

We start off by studying (33) for elements of $\mathfrak{A}$ of the form $B\left(F_{1}\right) \ldots B\left(F_{2 m}\right)$ for all $m \in \mathbb{N}$ and all $F_{1}, \ldots, F_{2 m} \in \mathfrak{H}$. Since the initial state $\omega_{\mathrm{d}}$ satisfies (32), we can write

$$
\omega_{\mathrm{d}}\left(\tau_{\gamma}^{t}\left[B\left(F_{1}\right) \ldots B\left(F_{2 m}\right)\right]\right)=\operatorname{pf}\left(\left[\Omega_{i, j}(t)\right]_{i, j=1}^{2 m}\right),
$$

where the following matrix, called the evolution matrix, is defined, for all $i, j \in\{1, \ldots, 2 m\}$ and all $t \in \mathbb{R}$, by

$$
\Omega_{i, j}(t):=\left(\mathrm{e}^{\mathrm{i} t H_{\gamma}} \Gamma F_{i}, S_{\mathrm{d}} \mathrm{e}^{\mathrm{i} t H_{\gamma}} F_{j}\right),
$$

and we used the fact from Remark 5 that $\left[\Gamma, \mathrm{e}^{\mathrm{i} t H_{\gamma}}\right]=0$.

Next, let us first concentrate on the limit (33) for 2-point functions.

(b) Spectral decomposition

Since we know from [10] that $1_{\mathrm{sc}}\left(H_{\gamma}\right)=0$, spectral theory yields the decomposition $1=$ $1_{\mathrm{ac}}\left(H_{\gamma}\right)+1_{\mathrm{pp}}\left(H_{\gamma}\right)$ which we insert into both arguments of the scalar product (39) between the propagators and the wave functions. The evolution matrix can thus be written as $\Omega_{i, j}(t)=$ $\Omega_{i, j}^{\mathrm{ac}}(t)+\Omega_{i, j}^{\mathrm{pp}}(t)+R_{i, j}^{(1)}(t)+R_{i, j}^{(2)}(t)$, where we set

$$
\begin{aligned}
& \Omega_{i, j}^{\mathrm{ac}}(t):=\left(\mathrm{e}^{\mathrm{i} t H_{\gamma}} 1_{\mathrm{ac}}\left(H_{\gamma}\right) \Gamma F_{i}, S_{\mathrm{d}} \mathrm{e}^{\mathrm{i} t H_{\gamma}} 1_{\mathrm{ac}}\left(H_{\gamma}\right) F_{j}\right), \\
& \Omega_{i, j}^{\mathrm{pp}}(t):=\left(\mathrm{e}^{\mathrm{i} t H_{\gamma}} 1_{\mathrm{pp}}\left(H_{\gamma}\right) \Gamma F_{i}, S_{\mathrm{d}} \mathrm{e}^{\mathrm{i} t H_{\gamma}} 1_{\mathrm{pp}}\left(H_{\gamma}\right) F_{j}\right),
\end{aligned}
$$

as well as $R_{i, j}^{(1)}(t):=\left(\mathrm{e}^{\mathrm{i} t H_{\gamma}} 1_{\mathrm{ac}}\left(H_{\gamma}\right) \Gamma F_{i}, S_{\mathrm{d}} \mathrm{e}^{\mathrm{i} t H_{\gamma}} 1_{\mathrm{pp}}\left(H_{\gamma}\right) F_{j}\right)$ and analogously for $R_{i, j}^{(2)}(t)$ with $1_{\mathrm{ac}}\left(H_{\gamma}\right)$ and $1_{\mathrm{pp}}\left(H_{\gamma}\right)$ interchanged.

(c) Large time ergodic mean

In order to study the limit (33), we will treat the foregoing terms separately.

As for the ac-term, using that the initial state is invariant under the decoupled dynamics, i.e., that $\left[S_{\mathrm{d}}, H_{\mathrm{d}}\right]=0$, we can write $\Omega_{i, j}^{\mathrm{ac}}(t)=\left(\mathrm{e}^{-\mathrm{i} t H_{\mathrm{d}}} \mathrm{e}^{\mathrm{i} t H_{\gamma}} 1_{\mathrm{ac}}\left(H_{\gamma}\right) \Gamma F_{i}, S_{\mathrm{d}} \mathrm{e}^{-\mathrm{i} t H_{\mathrm{d}}} \mathrm{e}^{\mathrm{i} t H_{\gamma}} 1_{\mathrm{ac}}\left(H_{\gamma}\right) F_{j}\right)$ and, hence, the large time ergodic mean from (33) becomes

$$
\lim _{t \rightarrow \infty} \Omega_{i, j}^{\mathrm{ac}}(t)=\left(\Gamma F_{i}, W_{\mathrm{d}, \gamma}^{*} S_{\mathrm{d}} W_{\mathrm{d}, \gamma} F_{j}\right),
$$

and we denote the right hand side of (42) by $\Omega_{i, j}^{\mathrm{ac}}$. 
Next, we also know from [10] that eig $\left(H_{\gamma}\right)$ is a finite set containing eigenvalues of finite multiplicity only (in the case at hand, the eigenvalues of $H_{\gamma}$ and their corresponding eigenfunctions can be computed explicitly but we do not need them to determine the heat flux in Theorem 22 below which is the main purpose of the present study). Hence, plugging $1_{\mathrm{pp}}\left(H_{\gamma}\right)=\sum_{e \in \mathrm{eig}\left(H_{\gamma}\right)} 1_{e}\left(H_{\gamma}\right)$ into $(41)$, and using that $\mathrm{e}^{\mathrm{i} t H_{\gamma}} 1_{e}\left(H_{\gamma}\right)=\mathrm{e}^{\mathrm{ite}} 1_{e}\left(H_{\gamma}\right)$, the large time ergodic mean of the pp-term reads

$$
\lim _{T \rightarrow \infty} \frac{1}{T} \int_{0}^{T} \mathrm{~d} t \Omega_{i, j}^{\mathrm{pp}}(t)=\sum_{e \in \operatorname{eig}\left(H_{\gamma}\right)}\left(\Gamma F_{i}, 1_{e}\left(H_{\gamma}\right) S_{\mathrm{d}} 1_{e}\left(H_{\gamma}\right) F_{j}\right) .
$$

Moreover, since $\left|R_{i, j}^{(1)}(t)\right| \leq\left\|1_{\mathrm{pp}}\left(H_{\gamma}\right) S_{\mathrm{d}} \mathrm{e}^{\mathrm{i} t H_{\gamma}} 1_{\mathrm{ac}}\left(H_{\gamma}\right) \Gamma F_{i}\right\|\left\|F_{j}\right\|$ due to the Cauchy-Schwarz inequality, and since $1_{\mathrm{pp}}\left(H_{\gamma}\right) \in \mathcal{L}^{0}(\mathfrak{H})$, we know from scattering theory that the right hand side of the foregoing estimate vanishes if $t \rightarrow \infty$. The term $R_{i, j}^{(2)}(t)$ is treated analogously.

We finally study the large time ergodic mean for observables of the form (38) and for general $A \in \mathfrak{A}$.

(d) Existence

Since the Pfaffian is a polynomial function of the entries of the matrix on which it acts and since $\Omega_{i, j}^{\mathrm{pp}}(t)$ is a trigonometric polynomial in $t$, the large time ergodic mean of $\mathrm{pf}\left(\left[\Omega_{i, j}^{\mathrm{ac}}+\right.\right.$ $\left.\Omega_{i, j}^{\mathrm{pp}}(t)\right]_{i, j=1}^{2 m}$ ) also exists (recall that, in general, the complex-valued functions on $\mathbb{R}$ which are almost periodic [in the sense of $\mathrm{H}$. Bohr, the brother of $\mathrm{N}$. Bohr] form an algebra with respect to the usual pointwise linear operations and multiplication, and the large time ergodic mean plays the role of a scalar product). Moreover, since, in addition, $\Omega_{i, j}(t)$ and $\Omega_{i, j}^{\mathrm{ac}}+\Omega_{i, j}^{\mathrm{pp}}(t)$ are both uniformly bounded in $t$, we have $\lim _{t \rightarrow \infty}\left|\operatorname{pf}\left(\left[\Omega_{i, j}(t)\right]_{i, j=1}^{2 m}\right)-\operatorname{pf}\left(\left[\Omega_{i, j}^{\mathrm{ac}}+\Omega_{i, j}^{\mathrm{pp}}(t)\right]_{i, j=1}^{2 m}\right)\right|=0$ which implies that

$$
\lim _{T \rightarrow \infty} \frac{1}{T} \int_{0}^{T} \mathrm{~d} t \omega_{\mathrm{d}}\left(\tau_{\gamma}^{t}\left[B\left(F_{1}\right) \ldots B\left(F_{2 m}\right)\right]\right)=\lim _{T \rightarrow \infty} \frac{1}{T} \int_{0}^{T} \mathrm{~d} t \mathrm{pf}\left(\left[\Omega_{i, j}^{\mathrm{ac}}+\Omega_{i, j}^{\mathrm{pp}}(t)\right]_{i, j=1}^{2 m}\right) .
$$

Finally, since $\mathfrak{A}$ is, by definition, the $C^{*}$-completion of the $*$-algebra generated by the identity 1 and the elements $B(F)$ and $B^{*}(F)$ satisfying (10) and (11) and since $\left|\omega_{\mathrm{d}}\left(\tau_{\gamma}^{t}(A)\right)\right| \leq$ $\|A\|$ for all $A \in \mathfrak{A}$, the existence of the limits (44) and the uniform convergence in $t$ of the ergodic mean for the approximant define the anisotropy NESS (33).

Hence, we arrive at the conclusion.

Remark 15 Since we know from [6] that, for the case at hand, the so-called XY 2-point operator $S:=W_{\mathrm{d}}^{*} S_{\mathrm{d}} W_{\mathrm{d}}$ is given by

$$
S=(1-s) \oplus \zeta s \zeta,
$$

where, in momentum space, $s \in \mathcal{L}(\mathfrak{h})$ acts through multiplication by the function $s_{\beta_{L}, \beta_{R}}$, where for all $\alpha, \beta \in \mathbb{R}$, we set

$$
s_{\alpha, \beta}(k):=1_{[0, \pi]}(k) \rho_{\alpha}(\cos (k))+1_{[-\pi, 0]}(k) \rho_{\beta}(\cos (k)),
$$


and since the chain rule for wave operators from scattering theory implies that $W_{\mathrm{d}, \gamma}=W_{\mathrm{d}} W_{\gamma}$, we can rewrite the first term on the right hand side of (37) in the form

$$
W_{\mathrm{d}, \gamma}^{*} S_{\mathrm{d}} W_{\mathrm{d}, \gamma}=W_{\gamma}^{*} S W_{\gamma}
$$

\section{Stationary scattering theory}

In this section, motivated by (47), we determine the action of the intermediate wave operator $W_{\gamma}$. To this end, we will make use of the so-called stationary approach to scattering theory (see, for example, [7] or [14] for a mathematical presentation of the theory and for the ingredients used in the proof of Proposition 16 below).

In the following, we switch to momentum space,

$$
\hat{\mathfrak{h}}:=L^{2}\left([-\pi, \pi] ; \frac{\mathrm{d} k}{2 \pi}\right),
$$

by means of the unitary Fourier transform $\mathfrak{f}: \mathfrak{h} \rightarrow \hat{\mathfrak{h}}$. The latter is defined, as usual, by $\mathfrak{f} f:=\sum_{x \in \mathbb{Z}} f(x) \mathrm{e}_{x}$, where the plane wave $\mathrm{e}_{x} \in \hat{\mathfrak{h}}$ is given by $\mathrm{e}_{x}(k):=\mathrm{e}^{\mathrm{i} k x}$ for all $x \in \mathbb{Z}$. Moreover, we extend it through $\mathfrak{F}:=\mathfrak{f} \oplus \mathfrak{f}$ to $\widehat{\mathfrak{H}}:=\hat{\mathfrak{h}} \oplus \hat{\mathfrak{h}}$ (the scalar products on $\hat{\mathfrak{h}}$ and $\widehat{\mathfrak{H}}$ are again both denoted by $(\cdot, \cdot))$. For all $a \in \mathcal{L}(\mathfrak{h})$ and all $A \in \mathcal{L}(\mathfrak{H})$, we set $\hat{a}:=\mathfrak{f} a \mathfrak{f}^{*} \in \mathcal{L}(\hat{\mathfrak{h}})$ and $\widehat{A}:=\mathfrak{F} A \mathfrak{F}^{*} \in \mathcal{L}(\widehat{\mathfrak{H}})$.

Proposition 16 (Wave operator) In momentum space $\widehat{\mathfrak{H}}$, the action of the wave operator $W_{\gamma}$ on completely localized wave functions is given, for all $x \in \mathbb{Z}$, by

$$
\widehat{W}_{\gamma} \mathrm{e}_{x} \oplus 0=\mathrm{e}_{x} \oplus 0-\frac{\gamma}{2}\left(w_{\gamma, x}^{(1)} \oplus 0+0 \oplus w_{\gamma, x}^{(2)}\right),
$$

where the functions $w_{\gamma, x}^{(1)}, w_{\gamma, x}^{(2)} \in \hat{\mathfrak{h}}$ are defined by

$$
\begin{aligned}
& w_{\gamma, x}^{(1)}:=w_{\gamma, x, a, a+1}^{(1)}+w_{\gamma, x, a+1, a}^{(1)}, \\
& w_{\gamma, x}^{(2)}:=w_{\gamma, x, a, a+1}^{(2)}-w_{\gamma, x, a+1, a}^{(2)},
\end{aligned}
$$

and, for all $a_{1}, a_{2} \in \mathbb{Z}$, we set

$$
\begin{aligned}
w_{\gamma, x, a_{1}, a_{2}}^{(1)}(k):= & \frac{\gamma}{2} \frac{\mathrm{e}_{a_{1}}(k)}{D_{\gamma}(k) \sin ^{2}(k)} \cdot \\
& \cdot\left[\mathrm{e}_{\left|x-a_{2}\right|+1}(|k|)+\mathrm{e}_{\left|x-a_{1}\right|}(|k|)+\mathrm{i} \frac{\gamma^{2}}{2} \frac{\mathrm{e}_{1}(|k|)}{\sin (|k|)}\left(\mathrm{e}_{\left|x-a_{2}\right|+1}(|k|)-\mathrm{e}_{\left|x-a_{1}\right|}(|k|)\right)\right], \\
w_{\gamma, x, a_{1}, a_{2}}^{(2)}(k):= & \mathrm{i}(-1)^{x-a_{1}} \frac{\mathrm{e}_{a_{1}}(k)}{\overline{D_{\gamma}(k)} \sin (|k|)} \cdot \\
& \cdot\left[\mathrm{e}_{\left|x-a_{2}\right|}(-|k|)+\frac{\gamma^{2}}{2} \frac{\mathrm{e}_{1}(-|k|)}{\sin ^{2}(k)}\left(\mathrm{e}_{\left|x-a_{2}\right|}(-|k|) \cos (k)-\mathrm{e}_{\left|x-a_{1}\right|}(-|k|)\right)\right]
\end{aligned}
$$


and also

$$
D_{\gamma}(k):=1+\gamma^{2} \frac{\mathrm{e}_{1}(|k|)}{\sin ^{2}(k)}\left(\cos (k)-\frac{\gamma^{2}}{4} \mathrm{e}_{1}(|k|)\right) .
$$

Remark 17 Note that $\left|D_{\gamma}(k)\right|^{2} \sin ^{4}(k)=\gamma^{4}\left(4-\gamma^{2}\right)^{2} / 16+\gamma^{2}\left[\left(2-\gamma^{2}\right)^{2}+\gamma^{2}\right] \sin ^{2}(k) / 2+(1-$ $\left.\gamma^{2}\right)^{2} \sin ^{4}(k)$. Hence, for $|\gamma|=2$, we have, in general, that $w_{\gamma, x, a_{1}, a_{2}}^{(i)} \notin \hat{\mathfrak{h}}$ but still $w_{\gamma, x}^{(i)} \in \hat{\mathfrak{h}}$.

Remark 18 Since $\Gamma \delta_{x} \oplus 0=0 \oplus \delta_{x}$, since $\left[\Gamma, \mathrm{e}^{-\mathrm{i} t H}\right]=\left[\Gamma, \mathrm{e}^{-\mathrm{i} t H_{\gamma}}\right]=0$ due to Remark 5, and since $\left[\Gamma, 1_{\mathrm{ac}}\left(H_{\gamma}\right)\right]=0$ due to the spectral theorem and the reflection invariance of the Lebesgue-Borel measure (implying that $\Gamma F$ belongs to the absolutely continuous subspace of $H_{\gamma}$ if $F$ does so), we get $\left[\Gamma, W_{\gamma}\right]=0$ and, hence, $\widehat{W}_{\gamma} 0 \oplus \mathrm{e}_{x}=\widehat{\Gamma} \widehat{W}_{\gamma} \mathrm{e}_{x} \oplus 0$ for all $x \in \mathbb{Z}$.

In the following, for all $a \in \mathcal{L}(\mathfrak{h})$ and all $A \in \mathcal{L}(\mathfrak{H})$, we denote by $r_{z}(a):=(a-z 1)^{-1}$ and $R_{z}(A):=(A-z 1)^{-1}$ the resolvents of $a$ and $A$ at points $z$ in the corresponding resolvent sets.

\section{Proof.}

\section{(a) Stationary approach}

The stationary approach to scattering theory expresses the wave operator as a weak abelian limit and subsequently transforms the time dependent propagators into time independent resolvents with the help of Parseval's identity, i.e., for all $F, G \in \mathfrak{H}$, we have

$$
\begin{aligned}
\left(G, W_{\gamma} F\right) & =\lim _{\varepsilon \rightarrow 0^{+}} 2 \varepsilon \int_{0}^{\infty} \mathrm{d} t \mathrm{e}^{-2 \varepsilon t}\left(G, \mathrm{e}^{-\mathrm{i} t H} \mathrm{e}^{\mathrm{i} t H_{\gamma}} 1_{\mathrm{ac}}\left(H_{\gamma}\right) F\right) \\
& =\lim _{\varepsilon \rightarrow 0^{+}} \int_{-\infty}^{\infty} \mathrm{d} e \frac{\varepsilon}{\pi}\left(R_{e-\mathrm{i} \varepsilon}(H) G, R_{e-\mathrm{i} \varepsilon}\left(H_{\gamma}\right) 1_{\mathrm{ac}}\left(H_{\gamma}\right) F\right) .
\end{aligned}
$$

(b) Interaction matrix

In order to compute the integrand in (55), we express the resolvent of $H_{\gamma}$ in terms of the resolvent of $H$ by means of the 2 nd resolvent identity, i.e., by $R_{e-\mathrm{i} \varepsilon}\left(H_{\gamma}\right)=R_{e-\mathrm{i} \varepsilon}(H)-$ $\gamma R_{e-\mathrm{i} \varepsilon}\left(H_{\gamma}\right) V R_{e-\mathrm{i} \varepsilon}(H)$. For all $e \in \mathbb{R}$, all $\varepsilon>0$, and all $F=f_{1} \oplus f_{2} \in \mathfrak{H}$, we then get

$$
\begin{aligned}
R_{e-\mathrm{i} \varepsilon}\left(H_{\gamma}\right) F=R_{e-\mathrm{i} \varepsilon}(H) F-\frac{\gamma}{2} R_{e-\mathrm{i} \varepsilon}\left(H_{\gamma}\right)( & {\left[r_{-e+\mathrm{i} \varepsilon}(h) f_{2}\right](a+1) \delta_{a} \oplus 0 } \\
& -\left[r_{-e+\mathrm{i} \varepsilon}(h) f_{2}\right](a) \delta_{a+1} \oplus 0 \\
& +\left[r_{e-\mathrm{i} \varepsilon}(h) f_{1}\right](a+1) 0 \oplus \delta_{a} \\
& \left.-\left[r_{e-\mathrm{i} \varepsilon}(h) f_{1}\right](a) 0 \oplus \delta_{a+1}\right),
\end{aligned}
$$

where we used that $R_{e-\mathrm{i} \varepsilon}(H)=r_{e-\mathrm{i} \varepsilon}(h) \oplus\left[-r_{-e+\mathrm{i} \varepsilon}(h)\right]$.

Next, we set $E_{1}:=\delta_{a} \oplus 0, E_{2}:=\delta_{a+1} \oplus 0, E_{3}:=0 \oplus \delta_{a}$, and $E_{4}:=0 \oplus \delta_{a+1}$, take the scalar product of (56) with $G \in \mathfrak{H}$ from the left, and set $\mu:=\left[\left(G, R_{e-\mathrm{i} \varepsilon}\left(H_{\gamma}\right) E_{i}\right)\right]_{i=1}^{4} \in \mathbb{C}^{4}$ and analogously for $\nu \in \mathbb{C}^{4}$ with $H_{\gamma}$ replaced by $H$. Then, plugging successively $F=E_{i}$ for all 
$i \in\{1,2,3,4\}$ into the terms from (56) and defining $\alpha_{e \pm \mathrm{i \varepsilon}}(x):=\left(\delta_{x}, r_{e \pm \mathrm{i} \varepsilon}(h) \delta_{0}\right)$ for all $e \in \mathbb{R}$, all $\varepsilon>0$, and all $x \in \mathbb{Z}$, we can compactly write the resulting four equations as $A_{\gamma, e-\mathrm{i} \varepsilon} \mu=\nu$, where the interaction matrix $A_{\gamma, e-\mathrm{i} \varepsilon} \in \mathbb{C}^{4 \times 4}$ is defined by

$$
A_{\gamma, e-\mathrm{i} \varepsilon}:=1+\frac{\gamma}{2}\left(\alpha_{e-\mathrm{i} \varepsilon}(1) \sigma_{1} \otimes \sigma_{3}+\alpha_{e-\mathrm{i} \varepsilon}(0) \sigma_{2} \otimes \sigma_{2}\right),
$$

and we made use of the symmetries (21), (22), and (23), implying, in particular, that $\alpha_{e-\mathrm{i} \varepsilon}(-x)$ $=\alpha_{e-\mathrm{i} \varepsilon}(x)$ and $\alpha_{-e+\mathrm{i} \varepsilon}(x)=(-1)^{x+1} \alpha_{e-\mathrm{i} \varepsilon}(x)$.

Moreover, since $\left(1+\gamma V R_{e-\mathrm{i} \varepsilon}(H)\right)\left(1-\gamma V R_{e-\mathrm{i} \varepsilon}\left(H_{\gamma}\right)\right)=1$ due to the 2 nd resolvent identity, since $A_{\gamma, e-\mathrm{i} \varepsilon}=\left[\left(E_{i},\left(1+\gamma V R_{e-\mathrm{i} \varepsilon}(H)\right) E_{j}\right)\right]_{i, j=1}^{4}$, and since the range of $V$ is spanned by $E_{1}$, $E_{2}, E_{3}$, and $E_{4}$, the interaction matrix is invertible. Hence, taking again the scalar product of (56) with $G \in \mathfrak{H}$ from the left and plugging $\mu$ into the resulting equation leads to

$$
\left(G, R_{e-\mathrm{i} \varepsilon}\left(H_{\gamma}\right) F\right)=\left(G, R_{e-\mathrm{i} \varepsilon}(H) F\right)-\frac{\gamma}{2} \sum_{i, j=1}^{4}\left(E_{i}^{\prime}, R_{e-\mathrm{i} \varepsilon}(H) F\right)\left[A_{\gamma, e-\mathrm{i} \varepsilon}^{-1}\right]_{i, j}\left(G, R_{e-\mathrm{i} \varepsilon}(H) E_{j}\right),
$$

where we set $E_{1}^{\prime}:=-E_{4}, E_{2}^{\prime}:=E_{3}, E_{3}^{\prime}:=E_{2}$, and $E_{4}^{\prime}:=-E_{1}$ (note that, using this notation, we can write $\left.V=\sum_{i=1}^{4}\left(E_{i}^{\prime}, \cdot\right) E_{i} / 2\right)$.

(c) Boundary values

We know from stationary scattering theory that, if the limit of $\varepsilon\left(R_{e-\mathrm{i} \varepsilon}(H) G, R_{e-\mathrm{i} \varepsilon}\left(H_{\gamma}\right) F\right) / \pi$ for $\varepsilon \rightarrow 0^{+}$exists for all $F, G \in \mathfrak{H}$ and almost all $e \in \mathbb{R}$ (where here and in the following, the set of full measure in $\mathbb{R}$ may depend on $F$ and $G$ ), the limit and the integration in (55) can be interchanged, the limit for $\varepsilon \rightarrow 0^{+}$of the integrand in (55) equals the limit of $\varepsilon\left(R_{e-\mathrm{i} \varepsilon}(H) G, R_{e-\mathrm{i} \varepsilon}\left(H_{\gamma}\right) F\right) / \pi$ for all $F, G \in \mathfrak{H}$ and almost all $e \in \mathbb{R}$, and the integral extends over $[-1,1]$ only since $\operatorname{spec}(H)=[-1,1]$.

In order to verify the existence in question, we use (58) and replace $G$ in (58) by the term $(\varepsilon / \pi) R_{e-\mathrm{i} \varepsilon}(H) G$. Since stationary scattering theory also guarantees the existence of the limits $\lim _{\varepsilon \rightarrow 0^{+}}\left(G, R_{e \pm \mathrm{i} \varepsilon}(H) F\right)$ for almost all $e \in \mathbb{R}$ (the existence argument holds for any Hamiltonian), and since $(\varepsilon / \pi) R_{e+\mathrm{i} \varepsilon}(H) R_{e-\mathrm{i} \varepsilon}(H)=\left(R_{e+\mathrm{i} \varepsilon}(H)-R_{e-\mathrm{i} \varepsilon}(H)\right) /(2 \pi \mathrm{i})$ due to the $1 \mathrm{st}$ resolvent identity, the limits of the $1 \mathrm{st}, 2 \mathrm{nd}$, and 4th term on the right hand side of (58) exist. As for the 3rd term, we know from [5] that, for all $e \in(-1,1)$ and all $x \in \mathbb{Z}$, the limit $\alpha_{e-\mathrm{i} 0}(x):=\lim _{\varepsilon \rightarrow 0^{+}} \alpha_{e-\mathrm{i} \varepsilon}(x)$ exists and has the form

$$
\alpha_{e-\mathrm{i} 0}(x)=-\mathrm{i} \frac{\left(e+\mathrm{i} \sqrt{1-e^{2}}\right)^{|x|}}{\sqrt{1-e^{2}}} .
$$

Since the limit from above of the expectation value of the resolvent of $H_{\gamma}$ (instead of $H$ ) also exists, and proceeding as in (b), we find that the matrix $A_{\gamma, e-\mathrm{i} 0}:=\lim _{\varepsilon \rightarrow 0^{+}} A_{\gamma, e-\mathrm{i} \varepsilon}$ is invertible, too. Since taking the inverse is a continuous operation, we also have that $\lim _{\varepsilon \rightarrow 0^{+}} A_{\gamma, e-\mathrm{i} \varepsilon}^{-1}=$ $A_{\gamma, e-\mathrm{i} 0}^{-1}$.

Moreover, for all $e \in(-1,1)$, we can compute the inverse and get

$$
A_{\gamma, e-\mathrm{i} 0}^{-1}=\frac{1}{\operatorname{det}\left(A_{\gamma, e-\mathrm{i} 0}\right)}\left(\beta_{\gamma, e}^{(1)} 1+\beta_{\gamma, e}^{(2)} \sigma_{3} \otimes \sigma_{1}+\beta_{\gamma, e}^{(3)} \sigma_{1} \otimes \sigma_{3}+\beta_{\gamma, e}^{(4)} \sigma_{2} \otimes \sigma_{2}\right),
$$


where we set

$$
\begin{aligned}
& \beta_{\gamma, e}^{(1)}:=1+\frac{\gamma^{2}}{2} \frac{e\left(e+\mathrm{i} \sqrt{1-e^{2}}\right)}{1-e^{2}}, \\
& \beta_{\gamma, e}^{(2)}:=-\frac{\gamma^{2}}{2} \frac{e+\mathrm{i} \sqrt{1-e^{2}}}{1-e^{2}} \\
& \beta_{\gamma, e}^{(3)}:=\mathrm{i} \frac{\gamma}{2} \frac{e+\mathrm{i} \sqrt{1-e^{2}}}{\sqrt{1-e^{2}}}\left(1+\mathrm{i} \frac{\gamma^{2}}{2} \frac{e+\mathrm{i} \sqrt{1-e^{2}}}{\sqrt{1-e^{2}}}\right), \\
& \beta_{\gamma, e}^{(4)}:=\mathrm{i} \frac{\gamma}{2} \frac{1}{\sqrt{1-e^{2}}}\left(1-\mathrm{i} \frac{\gamma^{2}}{2} \frac{e+\mathrm{i} \sqrt{1-e^{2}}}{\sqrt{1-e^{2}}}\right),
\end{aligned}
$$

and the determinant has the form

$$
\operatorname{det}\left(A_{\gamma, e-\mathrm{i} 0}\right)=1+\gamma^{2} \frac{e+\mathrm{i} \sqrt{1-e^{2}}}{1-e^{2}}\left(e-\frac{\gamma^{2}}{4}\left(e+\mathrm{i} \sqrt{1-e^{2}}\right)\right) .
$$

(d) Energy space representation

We next switch to energy space,

$$
\tilde{\mathfrak{h}}:=L^{2}\left([-1,1], \mathbb{C}^{2} ; \mathrm{d} e\right),
$$

by means of the unitary operator $\tilde{\mathfrak{f}}: \hat{\mathfrak{h}} \rightarrow \tilde{\mathfrak{h}}$ defined in [5] on the momentum space $\hat{\mathfrak{h}}$ as $(\tilde{\mathfrak{f}} \varphi)(e):=[\varphi(\arccos (e)), \varphi(-\arccos (e))] /\left(\sqrt{2 \pi} \sqrt[4]{1-e^{2}}\right)$. Since we want the XY Hamiltonian $H$ to become the operator acting through multiplication by the free variable in $\widetilde{\mathfrak{H}}:=\tilde{\mathfrak{h}} \oplus \tilde{\mathfrak{h}}$ (i.e., we want to make use of the multiplication operator version of the spectral theorem), we extend $\tilde{\mathfrak{f}}$ to $\widetilde{\mathfrak{F}}:=\tilde{\mathfrak{f}} \oplus \tilde{\theta} \tilde{\xi} \tilde{\mathfrak{f}}$ because of the 2nd factor in $H$, where, for all $a \in \mathcal{L}(\mathfrak{h})$ and all $A \in \mathcal{L}(\mathfrak{H})$, we set $\tilde{a}:=\tilde{\mathfrak{f}} \hat{a} \tilde{\mathfrak{f}}^{*} \in \mathcal{L}(\tilde{\mathfrak{h}})$ and $\widetilde{A}:=\widetilde{\mathfrak{F}} \widehat{A}_{\tilde{F}}^{*} \in \mathcal{L}(\widetilde{\mathfrak{H}})$, and we note that $(\tilde{\theta} \eta)(e)=\sigma_{1} \eta(e)$ and $(\tilde{\xi} \eta)(e)=\sigma_{1} \eta(-e)$ for all $\eta \in \tilde{\mathfrak{h}}$. Hence, since $(\hat{h} \varphi)(k)=\cos (k) \varphi(k)$ for all $\varphi \in \hat{\mathfrak{h}}$, the XY Hamiltonian $H$ indeed becomes the desired multiplication operator under conjugation with $\widetilde{\mathfrak{F}}$ because $(\tilde{h} \eta)(e)=e \eta(e)$ and $(\tilde{\theta} \tilde{\xi} \tilde{h} \tilde{\theta} \tilde{\xi} \eta)(e)=-e \eta(e)$ for all $\eta \in \tilde{\mathfrak{h}}$, where we used that $(\tilde{\theta} \tilde{\xi})^{*}=\tilde{\theta} \tilde{\xi}$ and that, for all $\eta=\left[\eta_{1}, \eta_{2}\right] \in \tilde{\mathfrak{h}}$, the adjoint of $\tilde{\mathfrak{f}}$ has the form

$$
\left(\tilde{\mathfrak{f}}^{*} \eta\right)(k)=\sqrt{2 \pi} \sqrt[4]{1-\cos ^{2}(k)}\left[1_{[0, \pi]}(k) \eta_{1}(\cos (k))+1_{[-\pi, 0]}(k) \eta_{2}(\cos (k))\right] .
$$

Furthermore, since the spectral core of $H$ equals $\operatorname{spec}(H)=[-1,1]$, stationary scattering theory also yields, for all $F=f_{1} \oplus f_{2} \in \mathfrak{H}$ and all $G=g_{1} \oplus g_{2} \in \mathfrak{H}$, that

$$
\lim _{\varepsilon \rightarrow 0^{+}} \frac{\varepsilon}{\pi}\left(R_{e-\mathrm{i} \varepsilon}(H) G, R_{e-\mathrm{i} \varepsilon}(H) F\right)=\left\langle\tilde{g}_{1}(e), \tilde{f}_{1}(e)\right\rangle+\left\langle\left(\tilde{\theta} \tilde{\xi} \tilde{g}_{2}\right)(e),\left(\tilde{\theta} \tilde{\xi} \tilde{f}_{2}\right)(e)\right\rangle
$$

where we set $\tilde{f}:=\tilde{\mathfrak{f}} \mathfrak{f} f$ for all $f \in \mathfrak{h}$, and $\langle\cdot, \cdot\rangle$ stands for the complex Euclidean scalar product on the constant fiber $\mathbb{C}^{2}$ of the direct integral (66). 
Now, plugging (58) into (55), commuting the limit and the integration, restricting the integration domain to $[-1,1]$ as discussed in (c), and substituting (68) into the resulting expression, we get

$$
\widetilde{W}_{\gamma} \widetilde{F}=\widetilde{F}-\frac{\gamma}{2} \sum_{i, j=1}^{4}\left(E_{i}^{\prime}, R_{\cdot-\mathrm{i} 0}(H) F\right)\left[A_{\gamma, \cdot-\mathrm{i} 0}^{-1}\right]_{i, j} \widetilde{E}_{j}
$$

where we set $\widetilde{F}:=\widetilde{\mathfrak{F} F} F$ for all $F \in \mathfrak{H}$ and $\left(E_{i}^{\prime}, R_{e-\mathrm{i} 0}(H) F\right):=\lim _{\varepsilon \rightarrow 0^{+}}\left(E_{i}^{\prime}, R_{e-\mathrm{i} \varepsilon}(H) F\right)$ exists as discussed in (c).

(e) Momentum space representation

Next, let $x \in \mathbb{Z}$ and plug $F=\delta_{x} \oplus 0$ into (69). Then, applying $\widetilde{\mathfrak{F}}=\tilde{\mathfrak{f}} \oplus \tilde{\theta} \tilde{\xi} \tilde{\mathfrak{f}}$ on both sides of (69) and noting that $\left(E_{1}^{\prime}, R_{{ }_{-\mathrm{i} 0} 0}(H) \delta_{x} \oplus 0\right)=\left(E_{2}^{\prime}, R_{{ }_{-\mathrm{i} 0}}(H) \delta_{x} \oplus 0\right)=0$ and that

$$
\begin{aligned}
& \left(E_{3}^{\prime}, R_{\cdot-\mathrm{i} 0}(H) \delta_{x} \oplus 0\right)=\alpha_{\cdot-\mathrm{i} 0}(x-(a+1)), \\
& \left(E_{4}^{\prime}, R_{\cdot-\mathrm{i} 0}(H) \delta_{x} \oplus 0\right)=-\alpha_{\cdot-\mathrm{i} 0}(x-a),
\end{aligned}
$$

the action, in momentum space $\widehat{\mathfrak{H}}$, of the intermediate wave operator $W_{\gamma}$ on completely localized wave functions becomes

$$
\begin{aligned}
\widehat{W}_{\gamma} \mathrm{e}_{x} \oplus 0 & =\mathrm{e}_{x} \oplus 0 \\
& -\frac{\gamma}{2} \sum_{j=1}^{4} \tilde{\mathfrak{f}}^{*} \oplus \tilde{\mathfrak{f}}^{*} \tilde{\theta} \tilde{\xi}\left[\left(\alpha_{\cdot-\mathrm{i} 0}(x-(a+1))\left[A_{\gamma,-\mathrm{i} 0}^{-1}\right]_{3, j}-\alpha_{{ }_{--\mathrm{i} 0}}(x-a)\left[A_{\gamma,--\mathrm{i} 0}^{-1}\right]_{4, j}\right) \widetilde{E}_{j}\right] .
\end{aligned}
$$

Finally, we plug (59) - (65) and (70) - (71) into (72) and use (67) and the fact that $\tilde{\delta}_{x}(e)=$ $\left[\left(e+\mathrm{i} \sqrt{1-e^{2}}\right)^{x},\left(e-\mathrm{i} \sqrt{1-e^{2}}\right)^{x}\right] /\left(\sqrt{2 \pi} \sqrt[4]{1-e^{2}}\right)$ for all $x \in \mathbb{Z}$.

Hence, we arrive at the conclusion.

\section{Entropy production rate}

In this section, we determine the expectation value in the anisotropy NESS of the energy flowing between the reservoirs through the sample system.

In the following, we will make use of the second quantization $b$ in the selfdual framework introduced in Definition 1(d).

\section{Definition 19 (Entropy production)}

(a) 1-particle energy current

The energy flow from the left reservoir into the sample is described by the 1-particle energy current observable $\Phi \in \mathcal{L}^{0}(\mathfrak{H})$ given by

$$
\Phi:=-\left.\frac{\mathrm{d}}{\mathrm{d} t}\right|_{t=0} \mathrm{e}^{\mathrm{i} t H_{\gamma}} H_{L} \mathrm{e}^{-\mathrm{i} t H_{\gamma}} .
$$


(b) Heat flux

The heat flux $J_{\gamma}$ is defined to be the NESS expectation value of the extensive energy current observable, i.e.,

$$
J_{\gamma}:=\omega_{\gamma}(b(\Phi))
$$

Moreover, the entropy production rate is given by $\sigma_{\gamma}:=\left(\beta_{R}-\beta_{L}\right) J_{\gamma}$.

Remark 20 Let us denote by $J_{\gamma, R}$ the NESS expectation value of the extensive energy current observable $b\left(\Phi_{R}\right)$ whose 1-particle observable $\Phi_{R}$ describes the energy flow from the right reservoir into the sample, i.e., $\Phi_{R}$ is defined as in (73) but with $H_{L}$ replaced by $H_{R}$. We thus get that the sum of the derivative (in the Banach space $\mathcal{L}(\mathfrak{H})$ ) from (73) and its analog for the right reservoir can be written as $\Phi+\Phi_{R}=-\mathrm{i}\left[H_{\gamma}, H_{R}+H_{L}\right]=\mathrm{i}\left[H_{\gamma}, Q\right]$, where we set $Q:=H_{\gamma}-\left(H_{R}+H_{R}\right)$, and we note that $Q \in \mathcal{L}^{0}(\mathfrak{H})$ because $Q=H_{S}+V_{\mathrm{d}}+\gamma V$. Here, as in Definition 8(b), $h_{S} \in \mathcal{L}(\mathfrak{h})$ is the 1-particle sample Hamiltonian defined by $h_{S}:=p_{S} h p_{S}$, and the orthogonal projection $p_{S} \in \mathcal{L}(\mathfrak{h})$ is given by $p_{S} f:=1_{\mathbb{Z}_{S}} f$ for all $f \in \mathfrak{h}$. Moreover, the lifting of $h_{S}$ to $\mathcal{L}(\mathfrak{H})$ is denoted by $H_{S}:=h_{S} \sigma_{3}$.

Since (12) and (31) imply that $\omega_{\gamma}(b(A))=-\operatorname{tr}\left(S_{\gamma} A\right)$ for all $A \in \mathcal{L}^{0}(\mathfrak{H})$ with $A=A^{*}$ and $\Gamma A \Gamma=-A$, where $\operatorname{tr}(\cdot)$ stands for the trace on $\mathcal{L}^{1}(\mathfrak{H})$, we get, with Remark 5 , that

$$
J_{\gamma}+J_{\gamma, R}=-i \operatorname{tr}\left(S_{\gamma}\left[H_{\gamma}, Q\right]\right)
$$

Since it follows from (33) that the anisotropy NESS $\omega_{\gamma}$ is invariant with respect to the anisotropy dynamics, i.e., since $\omega_{\gamma}\left(\tau_{\gamma}^{t}(A)\right)=\omega_{\gamma}(A)$ for all $t \in \mathbb{R}$ and all $A \in \mathfrak{A}$, we have $\left[S_{\gamma}, H_{\gamma}\right]=0$. Therefore, due to the cyclicity of the trace (i.e., $\operatorname{tr}(A B)=\operatorname{tr}(B A)$ for all $A \in \mathcal{L}^{1}(\mathfrak{H})$ and all $B \in \mathcal{L}(\mathfrak{H}))$, (75) implies that $J_{\gamma}+J_{\gamma, R}=0$, i.e., we obtain the first law of thermodynamics.

Remark 21 Due to Remark 20, the entropy production from Definition 19(b) has its usual form, i.e., we can write $\sigma_{\gamma}=-\left(\beta_{R} J_{\gamma, R}+\beta_{L} J_{\gamma}\right)$.

We now arrive at the main result of our study (see Figure 2).

Theorem 22 (Heat flux) For all $\gamma \in \mathbb{R}$, the heat flux has the form

$$
J_{\gamma}=\frac{1}{2} \int_{-\pi}^{\pi} \frac{\mathrm{d} k}{2 \pi} \sin (2|k|) \Delta(\cos (k))\left[1-\frac{P_{\gamma}(\sin (k))}{Q_{\gamma}(\sin (k))}\right],
$$

where we set $\Delta:=\rho_{\beta_{L}}-\rho_{\beta_{R}}$ and the even polynomials

$$
\begin{aligned}
P_{\gamma}(e) & :=\frac{b_{\gamma}}{2} e^{2}+c_{\gamma}, \\
Q_{\gamma}(e) & :=a_{\gamma} e^{4}+b_{\gamma} e^{2}+c_{\gamma},
\end{aligned}
$$




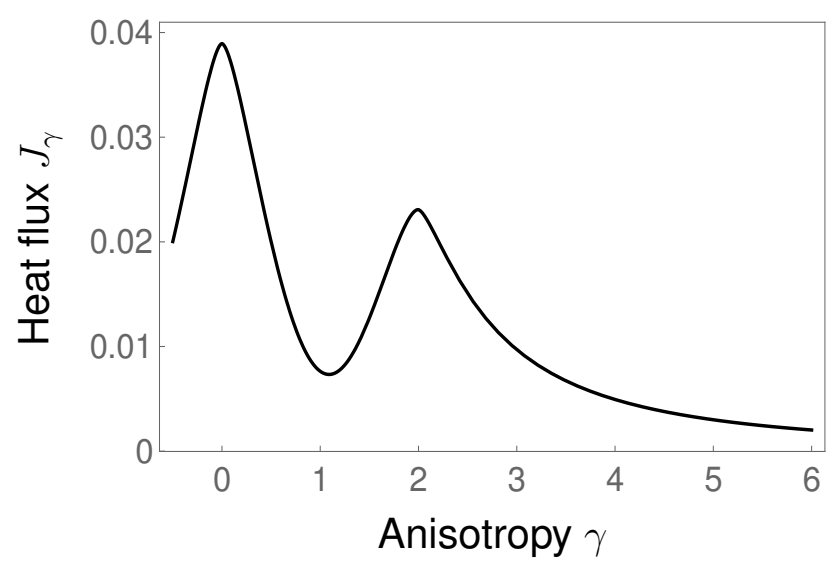

Figure 2: The heat flux $\gamma \mapsto J_{\gamma}$ for $\beta_{L}=1$ and $\beta_{R}=2$ (being an even function of the strength $\gamma$ of the anisotropy perturbation).

have the nonnegative coefficients

$$
\begin{aligned}
& a_{\gamma}:=\left(1-\gamma^{2}\right)^{2}, \\
& b_{\gamma}:=\frac{\gamma^{2}}{2}\left[\left(2-\gamma^{2}\right)^{2}+\gamma^{2}\right], \\
& c_{\gamma}:=\frac{\gamma^{4}}{16}\left(4-\gamma^{2}\right)^{2} .
\end{aligned}
$$

Remark 23 Since $1 /\left(1+\mathrm{e}^{x}\right)-1 /\left(1+\mathrm{e}^{y}\right)=\sinh ([y-x] / 2) /(\cosh ([y-x] / 2)+\cosh ([y+x] / 2))$ for all $x, y \in \mathbb{R}$, the difference of the Fermi-Dirac functions can be written as

$$
\Delta(e)=\frac{\sinh \left(\left[\beta_{R}-\beta_{L}\right] e / 2\right)}{\cosh \left(\left[\beta_{R}-\beta_{L}\right] e / 2\right)+\cosh \left(\left[\beta_{R}+\beta_{L}\right] e / 2\right)} .
$$

Remark 24 Note also that (76) is independent of the sample size $n$ and of the supporting sites $\{a, a+1\}$ of the local anisotropy perturbation.

Remark 25 In the light of (24), the values $\gamma=1$ and $\gamma=2$ correspond to a vanishing and, up to a global sign, an isotropic $\sigma_{2}$-contribution, respectively (see Figure 2). Moreover, Corollary 27 below yields that $J_{\gamma}>0$. The regularity of the heat flux function $\gamma \mapsto J_{\gamma}$ will be discussed elsewhere (see also [4]).

Proof.

(a) Flux structure

The 1-particle energy current observable (73) has the form

$$
\begin{aligned}
\Phi & =-\mathrm{i}\left[H_{\gamma}, H_{L}\right] \\
& =-\mathrm{i}\left[h, h_{L}\right] \sigma_{0}+\gamma\left\{v, h_{L}\right\} \sigma_{1} \\
& =\frac{1}{2} \operatorname{Im}\left[p_{-(n+2),-n}\right] \sigma_{0},
\end{aligned}
$$


where, in the last equality, we used that $v h_{\mathrm{L}}=h_{\mathrm{L}} v=0$ due to (6).

Since $\Phi=\Phi^{*} \in \mathcal{L}^{0}(\mathfrak{H})$ and $\Gamma \Phi \Gamma=-\Phi$, we have $\omega_{\gamma}(b(\Phi))=-\operatorname{tr}\left(S_{\gamma} \Phi\right)$ as in Remark 20. Hence, using (37) and (47), we write $J_{\gamma}=-\left(J_{\gamma, \mathrm{ac}}+J_{\gamma, \mathrm{pp}}\right)$ and we define

$$
\begin{aligned}
J_{\gamma, \text { ac }} & :=\operatorname{tr}\left(W_{\gamma}^{*} S W_{\gamma} \Phi\right), \\
J_{\gamma, \mathrm{pp}} & :=\sum_{e \in \operatorname{eig}\left(H_{\gamma}\right)} \operatorname{tr}\left(1_{e}\left(H_{\gamma}\right) S_{\mathrm{d}} 1_{e}\left(H_{\gamma}\right) \Phi\right) .
\end{aligned}
$$

(b) Term $J_{\gamma \text {,ac }}$

Using (83) and computing the trace (for example with respect to the orthonormal basis $\left\{\delta_{x} \oplus\right.$ $\left.0,0 \oplus \delta_{x}\right\}_{x \in \mathbb{Z}}$ of $\left.\mathfrak{H}\right)$, we get $J_{\gamma, \text { ac }}=J_{\gamma, \text { ac }}^{(1)}+J_{\gamma, \text { ac }}^{(2)}$, where we define

$$
\begin{aligned}
J_{\gamma, \mathrm{ac}}^{(1)} & :=\frac{1}{2} \operatorname{Im}\left[\left(W_{\gamma} \delta_{-(n+2)} \oplus 0, S W_{\gamma} \delta_{-n} \oplus 0\right)\right], \\
J_{\gamma, \mathrm{ac}}^{(2)} & :=\frac{1}{2} \operatorname{Im}\left[\left(W_{\gamma} 0 \oplus \delta_{-(n+2)}, S W_{\gamma} 0 \oplus \delta_{-n}\right)\right] .
\end{aligned}
$$

Let us first determine (86) by switching to momentum space and by specializing (49) (54) to the case $x=-n$ and $x=-(n+2)$. Using (6), we can write

$$
\begin{aligned}
& w_{\gamma,-n, a, a+1}^{(1)}(k)=\gamma \frac{\mathrm{e}_{a}(|k|+k) \mathrm{e}_{n+1}(|k|)}{D_{\gamma}(k) \sin ^{2}(k)}\left(\cos (k)-\frac{\gamma^{2}}{2} \mathrm{e}_{1}(|k|)\right), \\
& w_{\gamma,-n, a+1, a}^{(1)}(k)=\gamma \frac{\mathrm{e}_{a+1}(|k|+k) \mathrm{e}_{n}(|k|)}{D_{\gamma}(k) \sin ^{2}(k)},
\end{aligned}
$$

and also

$$
\begin{aligned}
& w_{\gamma,-n, a, a+1}^{(2)}(k)=\mathrm{i}(-1)^{n+a} \frac{\mathrm{e}_{a}(k-|k|) \mathrm{e}_{n+1}(-|k|)}{\overline{D_{\gamma}(k)} \sin ^{2}(k)}\left(\sin (|k|)-\mathrm{i} \frac{\gamma^{2}}{2} \mathrm{e}_{1}(-|k|)\right), \\
& w_{\gamma,-n, a+1, a}^{(2)}(k)=\mathrm{i}(-1)^{n+a} \frac{\mathrm{e}_{a}(k-|k|) \mathrm{e}_{n}(-|k|) \mathrm{e}_{1}(k)}{\overline{D_{\gamma}(k)} \sin ^{2}(k)}\left(\sin (|k|)+\mathrm{i} \frac{\gamma^{2}}{2} \mathrm{e}_{1}(-|k|)\right) .
\end{aligned}
$$

Moreover, for $\left(a_{1}, a_{2}\right) \in\{(a, a+1),(a+1, a)\}$, we get $w_{\gamma,-(n+2), a_{1}, a_{2}}^{(1)}(k)=\mathrm{e}_{2}(|k|) w_{\gamma,-n, a_{1}, a_{2}}^{(1)}(k)$ and $w_{\gamma,-(n+2), a_{1}, a_{2}}^{(2)}(k)=\mathrm{e}_{2}(-|k|) w_{\gamma,-n, a_{1}, a_{2}}^{(2)}(k)$. Next, we plug (88) - (91) and (45) into the scalar product on the right hand side of $(86)$ and note that $\mathfrak{f}(1-s) \mathfrak{f}^{*}$ and $\mathfrak{f} \zeta s \zeta \mathfrak{f}^{*}$ act through multiplication by the functions $s_{-\beta_{L},-\beta_{R}}$ and $s_{\beta_{R}, \beta_{L}}$ from (46), respectively. Decomposing the resulting expressions with respect to positive and negative momenta, taking the imaginary parts, regrouping with respect to the inverse temperatures, and using that, for all $\alpha, \beta \in \mathbb{R}$, we have $\rho_{-\alpha}-\rho_{-\beta}=-\left(\rho_{\alpha}-\rho_{\beta}\right)$ due to Remark 23 and $\rho_{-\alpha}(\cos (k)) \mapsto \rho_{\alpha}(\cos (k))$ if $k \mapsto k+\pi$ (the symmetry $\xi$ from Remark 6 ), a lengthy computation yields minus the right hand side of (76) divided by 2.

We next turn to (87). Using Remarks 9 and 18, we can write

$$
J_{\gamma, \mathrm{ac}}^{(2)}=J_{\gamma, \mathrm{ac}}^{(1)}-\frac{1}{2} \operatorname{Im}\left[\left(W_{\gamma} \delta_{-(n+2)} \oplus 0, W_{\gamma} \delta_{-n} \oplus 0\right)\right] .
$$


Since, for all $(\alpha, \beta) \in\left\{\left(-\beta_{L},-\beta_{R}\right),\left(\beta_{R}, \beta_{L}\right)\right\}$, we have $\lim _{\beta_{R} \rightarrow 0} \lim _{\beta_{L} \rightarrow 0} s_{\alpha, \beta}(k)=1 / 2$ and $\left|s_{\alpha, \beta}(k)\right| \leq 1$ for all $k \in[-\pi, \pi]$, Lebesgue's dominated convergence theorem implies, on one hand, that $\lim _{\beta_{R} \rightarrow 0} \lim _{\beta_{L} \rightarrow 0} J_{\gamma, \text { ac }}^{(1)}$ is equal to minus the second term on the right hand side of (92) divided by 2 . On the other hand, it also implies that $\lim _{\beta_{R} \rightarrow 0} \lim _{\beta_{L} \rightarrow 0} J_{\gamma, \text { ac }}^{(1)}=0$ since we know from above that $J_{\gamma, \text { ac }}^{(1)}$ is equal to minus the right hand side of (76) divided by 2 , since $\lim _{\beta_{R} \rightarrow 0} \lim _{\beta_{L} \rightarrow 0} \Delta(\cos (k))=0$ for all $k \in[-\pi, \pi]$, and since $|\Delta(\cos (k))| \leq 1 / 2$ due to (82) and $0 \leq 1-P_{\gamma}(\sin (k)) / Q_{\gamma}(\sin (k)) \leq 1$ for all $\gamma \in \mathbb{R}$ and all $k \in[-\pi, \pi]$ due to $(77)-(81)$. Hence, we find that $J_{\gamma, \text { ac }}=2 J_{\gamma, \text { ac }}^{(1)}$.

(c) Term $J_{\gamma, \mathrm{pp}}$

Using the cyclicity of the trace, all the summands on the right hand side of (85) can be written as $\operatorname{tr}\left(S_{\mathrm{d}} 1_{e}\left(H_{\gamma}\right) \Phi 1_{e}\left(H_{\gamma}\right)\right)$. Since $1_{e}\left(H_{\gamma}\right) \Phi 1_{e}\left(H_{\gamma}\right)=-\mathrm{i} 1_{e}\left(H_{\gamma}\right)\left[H_{\gamma}, H_{L}\right] 1_{e}\left(H_{\gamma}\right)=-\mathrm{i} 1_{e}\left(H_{\gamma}\right)\left(e H_{L}-\right.$ $\left.H_{L} e\right) 1_{e}\left(H_{\gamma}\right)=0$, we get $J_{\gamma, \mathrm{pp}}=0$.

Hence, we arrive at the conclusion.

Remark 26 Since scattering theory yields that $W_{\gamma}^{*} W_{\gamma}=1_{\mathrm{ac}}\left(H_{\gamma}\right)$, and since $1_{\mathrm{ac}}\left(H_{\gamma}\right)=1-$ $1_{\mathrm{pp}}\left(H_{\gamma}\right)$, the second term on the right hand side of (92) can also be determined using the eigenfunctions of $H_{\gamma}$.

Finally, we derive the strict positivity of the entropy production for the case at hand, i.e., in particular, we obtain the second law of thermodynamics.

Corollary 27 (Entropy production) For all $\gamma \in \mathbb{R}$, we have $0<\sigma_{\gamma} \leq\left(\beta_{R}-\beta_{L}\right) / 2$.

Proof.

(a) Case $\gamma \neq 0$

Due to the symmetry properties of the integrand in (76), the flux can be rewritten as an integral over the domain $[0, \pi / 2]$ on which all the factors of the integrand are nonnegative. Using, on this domain, the straightforward estimates $1-P_{\gamma}(\sin (k)) / Q_{\gamma}(\sin (k)) \geq b_{\gamma} \sin ^{2}(k) /\left[2\left(a_{\gamma}+\right.\right.$ $\left.\left.b_{\gamma}+c_{\gamma}\right)\right]$ and $\Delta(\cos (k)) \geq \sinh (\delta \cos (k)) / e_{0}$, where we set $e_{0}:=\cosh (\delta)+\cosh (\beta)$ with $\delta:=\left(\beta_{R}-\beta_{L}\right) / 2$ and $\beta:=\left(\beta_{R}+\beta_{L}\right) / 2$, and carrying out the remaining integration $d_{0}:=$ $\int_{0}^{\pi / 2} \mathrm{~d} k /(4 \pi) \sin (2 k) \sin ^{2}(k) \sinh (\delta \cos (k))=\left[\left(3+\delta^{2}\right) \sinh (\delta)-3 \delta \cosh (\delta)\right] /\left(\pi \delta^{4}\right)$, we get the lower bound $J_{\gamma} \geq 2 b_{\gamma} d_{0} /\left[\left(a_{\gamma}+b_{\gamma}+c_{\gamma}\right) e_{0}\right]>0$.

(b) Case $\gamma=0$

Since $P_{\gamma}(\sin (k))=0$ due to (80) and (81), we get the lower bound $J_{\gamma} \geq 4 d_{1} / e_{0}>0$ as in (a), where $d_{1}:=\int_{0}^{\pi / 2} \mathrm{~d} k /(4 \pi) \sin (2 k) \sinh (\delta \cos (k))=[\delta \cosh (\delta)-\sinh (\delta)] /\left(2 \pi \delta^{2}\right)$ (we already know from [6] that $J_{\gamma}>0$ in this case).

Hence, as soon as the system is truly out of equilibrium, i.e., if $\beta_{R}>\beta_{L}$, there exists a nonvanishing heat flux flowing through the sample from the left (hotter) to the right (colder) reservoir. Due to (4) and Definition 19(b), we also find that $\sigma_{\gamma}>0$. Moreover, the upper bound follows from the estimates after (92) in part (b) of the proof of Theorem 22.

Hence, we arrive at the conclusion. 
Remark 28 The upper bound can also be derived directly since $\left|\omega_{\gamma}(b(\Phi))\right| \leq\|b(\Phi)\|=$ $\|\Phi\|_{1} / 2$, where $\|\cdot\|_{1}$ stands for the trace norm, and we used [1] for the last equality.

\section{References}

[1] Araki H 1987 Bogoliubov automorphisms and Fock representations of canonical anticommutation relations Contemp. Math. 6223

[2] Araki H 1984 On the $X Y$-model on two-sided infinite chain Publ. RIMS Kyoto Univ. 20 277

[3] Araki H 1971 On quasifree states of CAR and Bogoliubov automorphisms Publ. RIMS Kyoto Univ. 6385

[4] Aschbacher W H 2016 On a quantum phase transition in a steady state out of equilibrium J. Phys. A: Math. Theor. 49415201

[5] Aschbacher W H 2011 Broken translation invariance in quasifree fermionic correlations out of equilibrium J. Funct. Anal. 2603429

[6] Aschbacher W H and Pillet C-A 2003 Non-equilibrium steady states of the XY chain J. Stat. Phys. 1121153

[7] Baumgärtel H and Wollenberg M 1983 Mathematical scattering theory (Birkhäuser)

[8] Bratteli O and Robinson D W 1987/1997 Operator algebras and quantum statistical mechanics 1/2 (Springer)

[9] Culvahouse J W, Schinke D P, and Pfortmiller L G 1969 Spin-spin interaction constants from the hyperfine structure of coupled ions Phys. Rev. 177454

[10] Hume L and Robinson D W 1986 Return to equilibrium in the $X Y$ model J. Stat. Phys. 44829

[11] Lieb E, Schultz T, and Mattis D 1961 Two soluble models of an antiferromagnetic chain Ann. Physics 16407

[12] Mikeska H-J and Kolezhuk A K 2004 One-dimensional magnetism in Schollwöck U, Richter J, Farnell D J J, and Bishop R F (Ed.) Quantum Magnetism Lect. Notes Phys. 6451 (Springer)

[13] Ruelle D 2001 Entropy production in quantum spin systems Commun. Math. Phys. 224 3

[14] Yafaev D R 1998 Mathematical scattering theory: general theory (AMS) 